\title{
Successful treatment of advanced thymic carcinoma with lymph node and pleural metastases: A case report
}

\author{
YOKO YAMAMOTO $^{1}$, KEN KODAMA $^{1}$, TOMOHIRO MANIWA ${ }^{1}$ and HIROKI KISHIMA ${ }^{2}$ \\ ${ }^{1}$ Department of Thoracic Surgery, Yao Municipal Hospital, Yao, Osaka 581-0069; \\ ${ }^{2}$ Department of Surgery, Kishima Hospital Main Division, Yao, Osaka 581-0853, Japan
}

Received May 24, 2016; Accepted July 4, 2016

DOI: $10.3892 / \mathrm{mco} .2016 .1006$

\begin{abstract}
Thymic carcinoma is a rare type of cancer that is more aggressive and is associated with a poorer prognosis compared with thymoma. No definitive clinical management of this disease has been established to date. This report describes the 20-year clinical course of a 50-year-old male patient who was successfully treated for initially diagnosed stage IVb thymic carcinoma. Thymo-thymectomy combined with resection of the surrounding organs was performed on December 1995 with curative intent (R0) after neoadjuvant chemoradiotherapy. From June 2008 until present, four pleural and subclavicular lymph node metastases have been treated with chemotherapy, radiotherapy and surgery, followed by low-dose oral steroid therapy. The patient has remained disease-free for the last 20 months since the initiation of prednisolone treatment. Thus, multidisciplinary treatment may achieve long-term survival in patients with stage IVb thymic carcinoma.
\end{abstract}

\section{Introduction}

Thymic carcinoma is a rare type of cancer, which differs from thymoma regarding its aggressive clinical behavior. The majority of patients with thymic carcinoma present at an advanced stage and have a poor prognosis $(1,2)$. However, a proportion of the patients who achieve long-term survival develop disease relapse and are candidates for salvage therapy, such as re-resection, radiotherapy, or other modalities (3-5). We herein present the 20-year clinical course of a patient initially diagnosed with stage IVb thymic carcinoma that developed bidirectional metastases toward the pleural and lymph nodes, which were successfully treated with repeated chemotherapy, surgery and radiotherapy, followed by low-dose steroid therapy.

Correspondence to: Dr Yoko Yamamoto, Department of Thoracic Surgery, Yao Municipal Hospital, 1-3-1 Ryuge-Cho, Yao, Osaka 581-0069, Japan

E-mail: yokes615@yahoo.co.jp

Key words: thymic carcinoma, stage IVb, surgery, long-term survival

\section{Case report}

A 50-year-old man visited a regional hospital 20 years prior with hemoptysis and a febrile state. A chest X-ray revealed a $6 \times 5-\mathrm{cm}$ solid mass in the hilum of the left lung, directly invading the mediastinum, with left phrenic nerve palsy (Fig. 1). Squamous cell carcinoma was diagnosed by percutaneous needle biopsy. Based on the clinical diagnosis of T4NOM0 lung cancer, sequential chemoradiotherapy with two cycles of mytomycin $\mathrm{C}$, vindecine and cisplatin (MVC regimen), and radiotherapy of 60 Gy was administered. The patient was subsequently referred to the Osaka Medical Center for surgery and he underwent en bloc resection of the left upper lobe, mediastinal lymph nodes, left brachiocephalic vein, pericardium and thymus via a left hemiclamshell approach. The left brachiocephalic vein was reconstructed using a Gore-Tex graft (Gore Medical, Flagstaff, AZ, USA). As it was included in the radiation field, the bronchial stump was covered with the omentum major. The final pathological diagnosis was thymic carcinoma with mediastinal lymph node metastasis (Masaoka stage IVb). The histological response to chemoradiation therapy was poor (Ef-1).

The postoperative course was uneventful. Eleven years after the initial operation, the patient underwent high anterior resection for colon cancer at another hospital. Twelve years and six months after the initial operation, a heterogeneous mass with a maximal diameter of $10 \mathrm{~cm}$ in close contact with the 12th thoracic to the 2nd lumbar vertebrae was identified on follow-up computed tomography (CT). As no definitive diagnosis was made by needle biopsy, oral tegafur-uracil/leucovorin was administered and a partial response was obtained. Sixteen years after the initial operation, at 66 years of age, the patient was referred to the Yao Municipal Hospital (Yao, Japan) for investigation of an acute-onset left back pain. Laboratory examinations revealed results within normal limits, including tumor markers. A CT scan revealed a re-growing left paravertebral mass that extended into the 1st intervertebral foramen (Fig. 2). Magnetic resonance imaging demonstrated that the mass exhibited a low signal intensity on T1-weighted images and a high signal intensity on T2-weighted and diffusion-weighted images. At that time, pleural recurrence of the thymic carcinoma at the costodiaphragmatic recess or a neurogenic tumor was suspected, and surgery was performed for the purpose of a definitive diagnosis and treatment. Intraoperatively, a mass sized $8.5 \times 4.0 \times 7.0 \mathrm{~cm}$ was 
identified at the bottom of the left chest cavity, in close contact with the 12th thoracic and 1st lumbar vertebrae, and the 11th and 12 th ribs. Although the mass was resected with the surrounding connective tissue, including the ribs, the resection was incomplete due to the invasive nature of the mass at the intervertebral foramen. Pathologically, the mass was composed of highly atypical epithelioid cells with nuclear atypia and eosinophilic cytoplasm in a fibrotic stroma. The Ki-67 index was $27 \%$, with positive reactivity for CD5 and cytokeratin AE1/AE3, and negative reactivity for c-Kit, synaptophysin, chromogranin and thyroid transcription factor 1 . The final diagnosis of the mass was pleural dissemination of the thymic carcinoma.

After the operation, $46 \mathrm{~Gy} / 23$ fr of adjuvant radiation therapy was delivered to the resection margin site, including the intervertebral foramen. The postoperative course was uneventful and the left back pain disappeared.

Twenty months following metastasectomy, positron emission tomography/CT revealed a left subclavicular lymph node metastasis and three new pleural metastases (Fig. 3). The patient received systemic chemotherapy comprising three cycles of carboplatin (area under the curve 5 on day 1 , every 21 days) and paclitaxel $\left(200 \mathrm{mg} / \mathrm{m}^{2}\right.$ on day 1 , every 21 days). The effects were minimal and the response was classified as stable disease. Therefore, radiation therapy was delivered (46 Gy/23 fr) to all four lesions. The chest CT showed that the left subclavicular lymph node metastasis had markedly shrunk, but the pleural metastases showed no marked changes. No new lesions appeared over the next 11 months. Therefore, complete excision of the three pleural masses was conducted with clearance of all the intervening connective tissue, combined with partial resections of the diaphragm and lung, 18 years after the initial operation.

One month after surgery, the patient developed eosinophilic pneumonia in the left lung. Oral administration of prednisolone (30 mg/day) was initiated to control the pneumonia. Two months after surgery, the dose was tapered by $5 \mathrm{mg} /$ day every 2 weeks. The eosinophilic pneumonia, as well as the patient's general condition, including wound pain, markedly improved, and he requested continuous daily oral administration of $5 \mathrm{mg}$ prednisolone.

The patient is currently disease-free and has received low-dose prednisolone for 20 months.

\section{Discussion}

Thymic epithelial neoplasms are a relatively rare type of cancer, with an annual incidence of $0.15 / 100,000$ person-years (6). The 2004 WHO classification clearly separates thymic carcinoma from thymoma (7). Thymic carcinoma accounts for $<1-4 \%$ of thymic epithelial neoplasms. A previous report indicated that the incidence of nodal metastases in patients with thymic carcinoma was $26.8 \%$ (1). It has been confirmed by several studies that patients with thymic carcinoma receiving complete resection (R0) exhibit a more favorable long-term survival compared with those who undergo incomplete resection or do not receive surgery $(1,5)$.

There is currently no standard guideline for the management of recurrent thymic carcinoma. Wright et al reported that the recurrence rate of type $\mathrm{C}$ thymoma (thymic carcinoma) is $50 \%$ (8). The majority of recurrences appear as pleural dissemination,

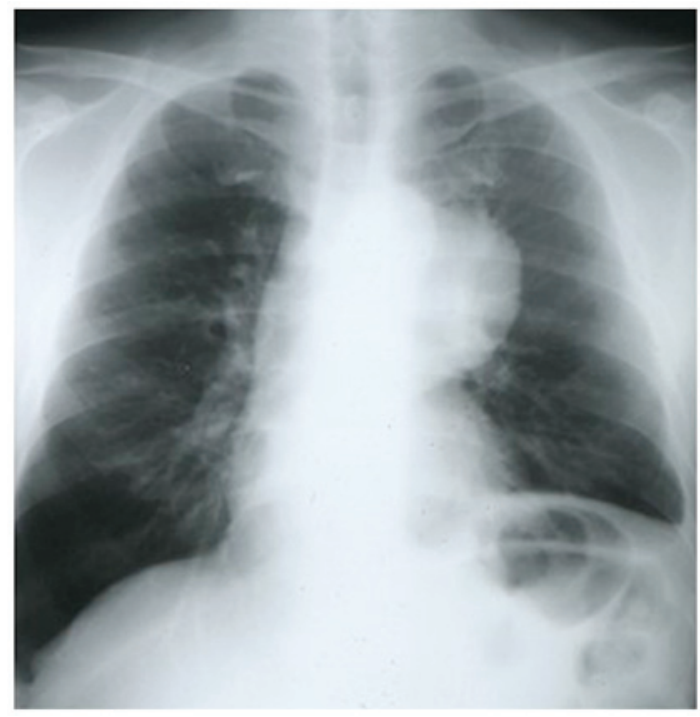

Figure 1. Chest X-ray showing a $6 \times 5-\mathrm{cm}$ solid mass in the hilum of the left lung, directly invading the mediastinum, with left phrenic nerve palsy.

A

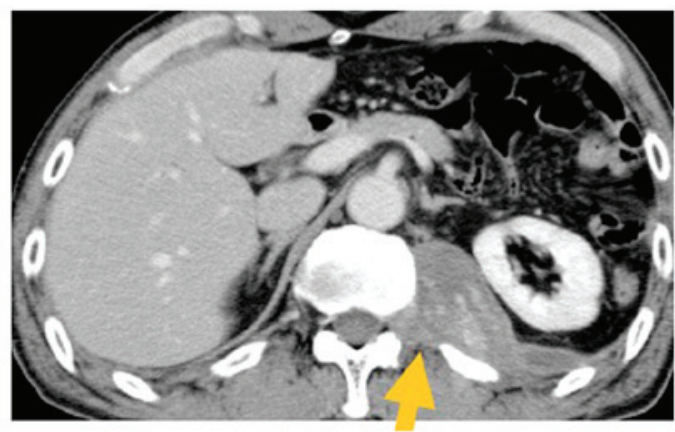

B

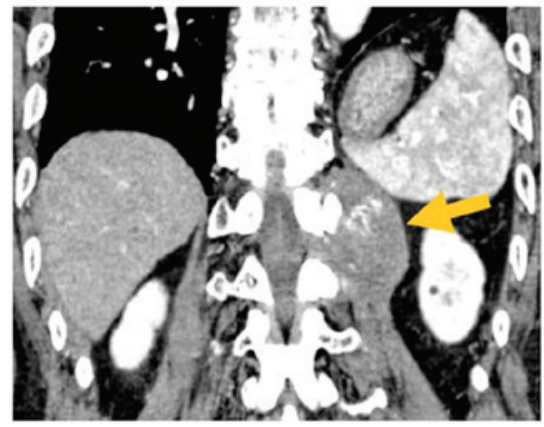

Figure 2. Computed tomography scan showing a left paravertebral mass extending into the 1st intervertebral foramen. (A) Horizontal axis and (B) coronal axis.

mediastinal relapse, or both. Most thymic carcinomas present at an advanced stage and have a high frequency of relapse, even after complete resection, which indicates the need for a multidisciplinary therapeutic approach (5). Multidisciplinary therapy, including re-excision, radiotherapy and chemotherapy, is recommended. However, the impact of these modalities on the clinical outcome is unclear. Surgery may be a reasonable option in patients with localized recurrence that can be completely resected $(3,4)$. Radiotherapy may improve local control and also confer some survival benefits $(5,9)$. Chemotherapy is based on low-level evidence: Platinum-based doublet chemotherapy has achieved some beneficial effects (10). 


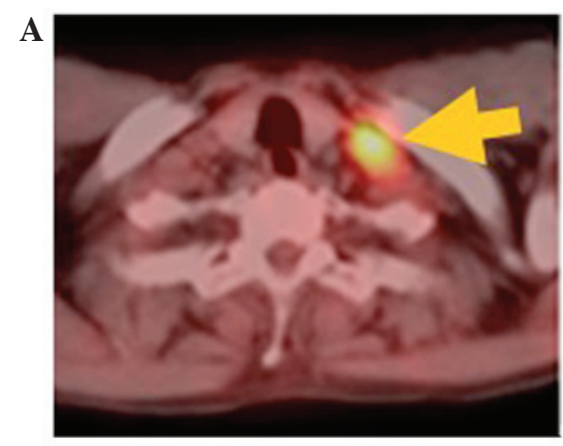

B
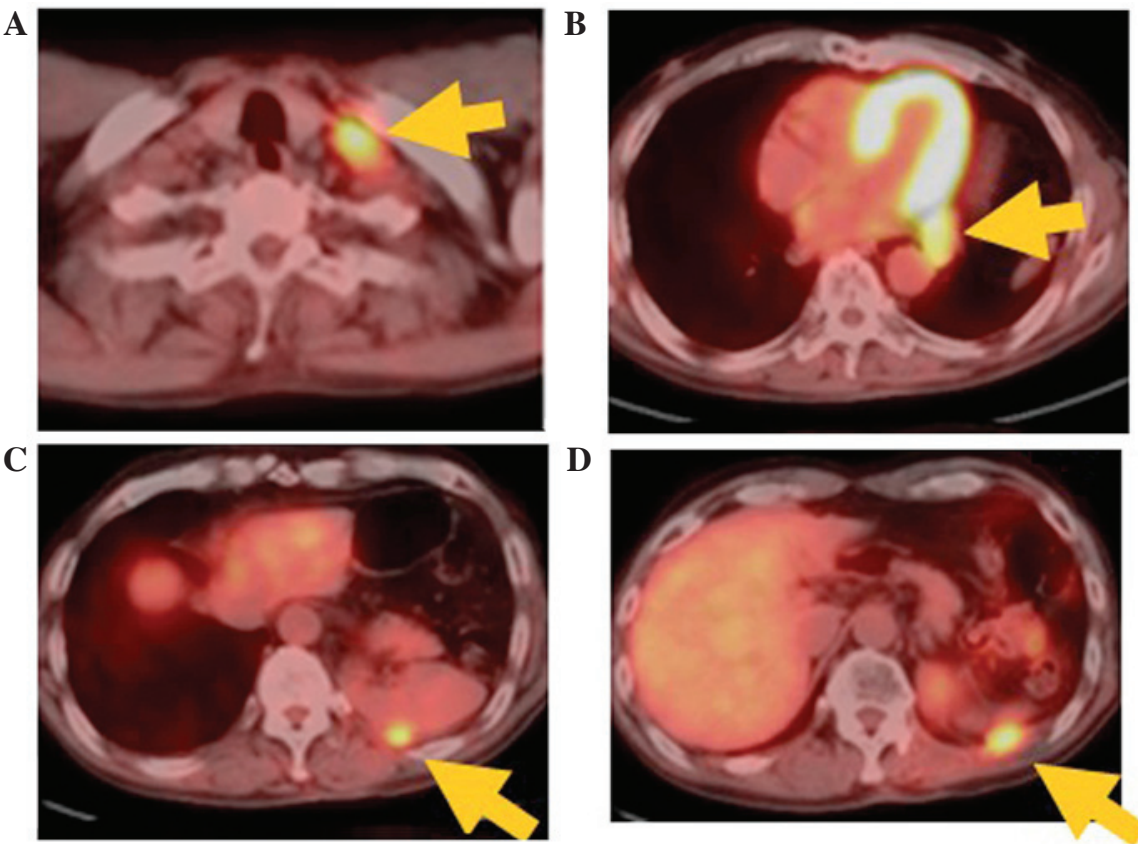

Figure 3. Positron emission tomography/computed tomography scan showing three pleural recurrences and a left subclavicular lymph node metastasis.

According to a Japanese nationwide database study, stage IVb thymic carcinoma patients showed a relatively favorable survival if they underwent $\mathrm{R} 0$ resection for locoregional nodal and pulmonary metastases, with 37\% 10-year recurrence-free survival and 39\% 10-year overall survival (5). We were able to perform R0 resection for the primary site in our patient, with combined resection of the left upper lobe of the lung, left brachiocephalic vein and pericardium after chemoradiotherapy. There has been no recurrence at the primary site for 20 years. Repeated metastases at the bottom of the left chest cavity and left subclavicular lymph nodes were controlled with chemotherapy, surgery and radiotherapy. Over the last 20 months, we have continued to administer low-dose oral prednisolone (5 mg/day), without recurrence.

Glucocorticoids are often administered to patients with thymomas to treat associated symptoms of myasthenia gravis, rather than the tumor itself. Funakoshi et al reported that thymoma and thymic carcinoma express glucocorticoid receptors, and glucocorticoids may directly affect tumor cells in addition to affecting associated lymphocytes (11). There have been a few reports describing the marked clinical response of refractory thymoma, but not of thymic carcinoma (12). Although the glucocorticoid receptor expression of tumor cells was not investigated in our patient, low-dose prednisolone, which was administered to treat eosinophilic pneumonia, may promote the response of resistant cells to multidisciplinary treatment and maintain a disease-free status in our patient.

In conclusion, the multidisciplinary treatment described herein may achieve long-term survival in patients with stage IVb thymic carcinoma.

\section{References}

1. Kondo K and Monden Y: Therapy for thymic epithelial tumors: A clinical study of 1320 patients from Japan. Ann Thorac Surg 76: 878-885, 2003.
2. Suster S and Rosai J: Thymic carcinoma. A clinicopathologic study of 60 cases. Cancer 67: 1025-1032, 1991.

3. Toyokawa G, Taguchi K, Kojo M, Toyozawa R, Inamasu E, Morodomi Y, Shiraishi Y, Takenaka T, Hirai F, Yamaguchi M, et al: Recurrence of thymic neuroendocrine carcinoma 24 year after total excision: A case report. Oncol Lett 6: 147-149, 2013.

4. Bott MJ, Wang H, Travis W, Riely GJ, Bains M, Downey R, Rusch V and Huang J: Management and outcomes of relapse after treatment of thymoma and thymic carcinoma. Ann Thorac Surg 92: 1984-1992, 2011.

5. Hishida T, Nomura S, Yano M, Asamura H, Yamashita M, Ohde Y, Kondo K, Date H, Okumura M and Nagai K; Japanese Association for Research on the Thymus (JART): Long-term outcome and prognostic factors of surgically treated thymic carcinoma: Results of 306 cases from a Japanese nationwide database study. Eur J Cardiothorac Surg 49: 835-841, 2016.

6. Ebgels EA and Pfeiffer RM: Malignant thymoma in the United states: Demographic patterns in incidence and associations with subsequent malignancies. Int J Cancer 105: 546-551, 2003.

7. Travis WD, Brambilla E, Muller-Hermelink HK and Hariis CC: World health organization classification of tumors. Pathology and genetics of tumors of the lung, pleura, thymus and heart. Chapter. 3rd edition. IARC Press, Lyon, pp145-pp975, 2004.

8. Wright CD, Wain JC, Wong DR, Donahue DM, Gaissert HA, Grillo HC and Mathisen DJ: Predictors of recurrence in thymic tumors: Importance of invasion, world health organization histology and size. J Thorac Cardiovasc Surg 130: pp1413-1421, 2005.

9. Ogawa K, Toita T, Uno T, Fuwa N, Kakinohana Y, Kamata M, Koja K, Kinjo T, Adachi G and Murayama S: Treatment and prognosis of thymic carcinoma: A retrospective analysis of 40 cases. Cancer 94: 3115-3119, 2002.

10. Nakamura Y, Kunito H, Kubota K, Sekine I, Shinkai T, Tamura T, Kodama T, Sumi M, Kohno S and Saijo N: Platinum-based chemotherapy with or without thoracic radiation therapy in patients with unresectable thymic carcinoma. Jpn J Clin Oncol 30: 385-388, 2000.

11. Funakoshi Y, Shiono H, Inoue M, Kadota Y, Ohta M, Matsuda H, Okumura M and Eimoto T: Glucocorticoids induce G1 cell cycle arrest in human neoplastic thymic epithelial cells. K Cancer Res Clin Oncol 131: 314-322, 2005.

12. Kodama K, Doi S, Higashiyama M, Yokouchi H, Yasuda T and Funai H: Dramatic response of postthymomectomy myasthenia gravis with multiple lung nodules to corticosteroids. Ann Thorac Surg 64: 555-557, 1997. 